\section{Effective Hospital Waste Classification to Overcome Occupational Health Issues and Reduce Waste Disposal Cost}

To the Editor-Medical and healthcare institutions can be the source of life-saving medical interventions, but they also play a significant role as a vital energy hogs. At the same time, healthcare and medical institutions conduct different kinds of therapeutic activities that result in the production of infectious waste, sharp objects, radioactive waste, and chemical materials. ${ }^{1}$ Research conducted by Yoan Kagoma, a medical director, showed that proper waste segregation is the single most effective cost-saving measure; therefore, he has suggested that all hospitals should compulsorily put this into practice. ${ }^{2}$ In addition, a hospital should reduce its ecological footprint by practicing comprehensive medical waste segregation and integrating "reduce, reuse, recycle" concepts into its medical waste management system. ${ }^{3}$ Moreover, without designated medical waste segregation, enormous waste from medical institutions, especially operating rooms, is classified as "biohazard waste." Thus, biohazard waste is a category of waste that requires expensive handling, packaging, and incineration, which have been estimated to cost 8 times more per tonne than solid waste in the disposal process (Figure 1).

The significant waste that has been left out from the classification is recyclable waste, which is the crucial aspect in

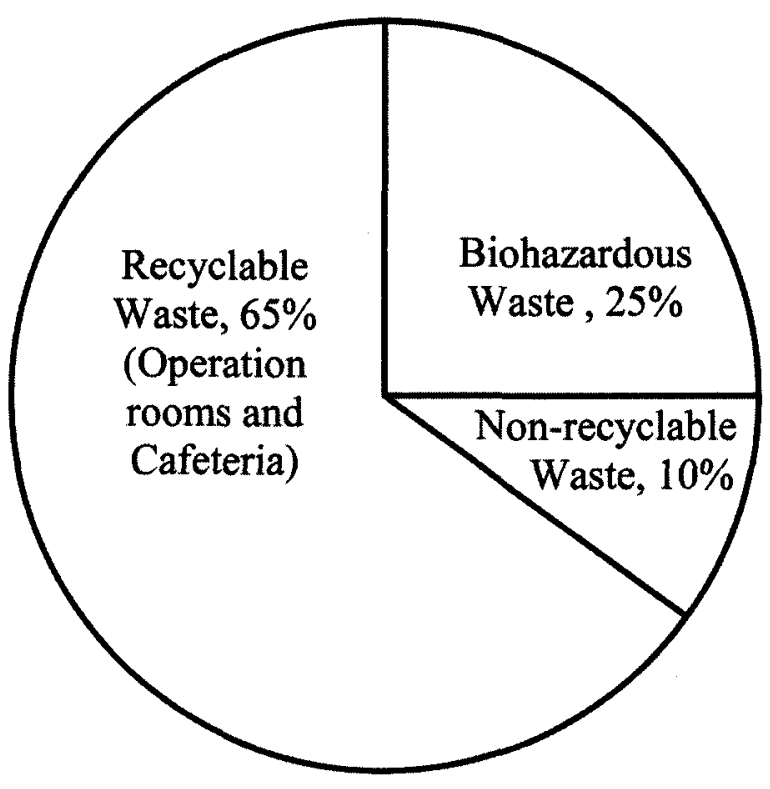

FIGURE 1. Distribution of the main categories of waste produced by medical institutions. reducing waste management costs. In medical waste, blood bags and fluid (intravenous) bags are the common items that contain furans and dioxins. The main issue here is that, instead of recycling them, medical institutions burn plastic and paper products using a medical waste incinerator. Thus, during incineration dioxins and furans are formed and released into the air. In addition, for many years hospitals have been segregating medical trash like used hypodermic needles from other waste. Although the main objective of this separation is to avoid the health risk it imposes, this method of separation has been useful in the process of recycling used needles.

At the same time, hospitals are now looking for disposable medical devices that can be sterilized and recycled to reduce the cost to the patient by $40 \%-60 \%$. In the meantime, to avoid worries about the increased risk of infection or disease transmission, a study was done in 2008 by the Government Accounting Office. It has been documented that the use of sterilized, recycled medical supplies did not increase the risk of disease transmission or decrease patient safety at all. In addition, operating rooms have become the main target of administrators as they are looking to reduce waste. Singleuse supplies will normally be opened and kept on-hand for potential use during a procedure. The other items in the same package may not be used and must be discarded.

Therefore, to overcome all the mentioned weaknesses in the existing classification, a new classification of medical waste has been developed. The following 8 classes are suggested through this study: microbiological waste, pathological waste, sharps waste, pharmaceutical waste, chemical waste, radioactive waste, nonrecyclable waste, and recyclable waste.

Surveys have shown that an appropriate method of identifying and segregating waste is to sort the waste by means of color codes. The deficient element in the existing color coding system for medical waste segregation is the unavailability of different containers for the subdivision of medical waste ( 8 classes). The color coding system that has been proposed in this study after a depth analysis is shown in Table 1. All waste must be collected at the point of generation, and

TABLE 1. Suggested Color Codes for Different Types of Medical Waste

\begin{tabular}{ll}
\hline Color code & \multicolumn{1}{c}{ Medical waste } \\
\hline Red & Microbiological waste \\
Yellow & $\begin{array}{l}\text { Pathological waste } \\
\text { Blue }\end{array}$ \\
& $\begin{array}{l}\text { Sharps waste (in leak-proof and puncture- } \\
\text { resistant containers) }\end{array}$ \\
Brown & Pharmaceutical waste \\
Orange & Chemical waste \\
Silver & Radioactive waste \\
Black & Nonrecyclable waste \\
Green & Recyclable waste \\
\hline
\end{tabular}


all containers must bear international symbols with appropriate wording.

In conclusion, regulated medical waste volumes could be reduced by $40 \%$ and violations of segregation could be reduced by $90 \%$ if best environmental practices such as this new classification of hospital waste were adopted, and in turn it could reduce the cost of waste disposal, given that every hospital produces about $80 \%$ of recyclable waste. Because of improper classification of waste, they are considered to be hazardous and more money is spent on their disposal every year. By following this proper classification system and its disposal method, we can economically spend money on hospital waste disposal.

\section{ACKNOWLEDGMENTS}

We are very grateful for the research grant provided by the Science Fund, Ministry of Science, Technology, and Innovation (MOSTI), and for its support and publication of the paper that has resulted in this letter.

Potential conflicts of interest. All authors report no conflicts of interest relevant to this article. All authors submitted the ICMJE Form for Disclosure of Potential Conflicts of Interest, and the conflicts that the editors consider relevant to this article are disclosed here.

V. Ramani Bai, $\mathrm{PhD} ;^{1}$ G. Vanitha, $\mathrm{PhD}{ }^{1}$ A. R. Zainal Ariff, BDS, MSc, FFDRCS, FDSRCS ${ }^{2}$

Affiliations: 1. Department of Civil Engineering, University of Nottingham, Malaysia Campus, Semenyih, Malaysia; 2. Department of Oral and Maxillofacial Surgery, Universiti Malaya, Kuala Lumpur, Malaysia.

Address correspondence to V. Ramani Bai, $\mathrm{PhD}$, Department of Civil Engineering, University of Nottingham (M) Campus, Mn Broga, Semenyih, Selangor 43500, Malaysia (vramanibai@gmail.com).

Infect Control Hosp Epidemiol 2013;34(11):1234-1235

(C) 2013 by The Society for Healthcare Epidemiology of America. All rights reserved. 0899-823X/2013/3411-0021\$15.00. DOI: $10.1086 / 673461$

\section{REFERENCES}

1. Coote $A$. What health services could do about climate change. BMJ 2006;332:1343-1344.

2. Kagoma Y, Stall N, Rubinstein E, Naudie D. People, planet and profits: the case for greening operating rooms. Can Med Assoc J 2012;184:1905-1911.

3. Kralj D, Markic M. Building materials reuse and recycle. WSEAS Trans Environ Dev 2008;4:409-418.

\section{Associations between Carbapenem Use, Carbapenem-Resistant Pseudomonas aeruginosa, and Carbapenem-Resistant Acinetobacter baumannii}

To the Editor-Appropriate antimicrobial therapy is associated with reduction in mortality., Empiric antimicrobial therapy has led to the increased use of carbapenems for initial broad-spectrum treatment of nosocomial infections worldwide. ${ }^{1,2} \mathrm{~A}$ few studies have suggested that carbapenems have a differential impact on gut ecology, defined as the emergence of carbapenem-resistant Acenitobacter baumannii (CRAB) and carbapenem-resistant $P$ seudomonas aeruginosa (CRPA). ${ }^{3,4}$ In 2 studies, doripenem was less likely to select for CRPA yet was similar in gut-selection capacity to imipenem-cilastatin and meropenem for CRAB ${ }^{3,4}$ Limited data are available to inform on the relationship between carbapenem use and the prevalence of CRAB and CRPA. We therefore conducted a study to assess the association between carbapenem consumption and the change in prevalence of CRAB and CRPA in a single hospital over an 11-month period.

From July 1 to September 30, 2012 (period 1), the increase in the incidence of CRPA in both surgical and medical units was defined as an outbreak at Thammasat University Hospital and associated with increased empiric doripenem and fosfomycin use; $C R A B$ estimates remained stable during this interval (Figure 1). The incidence of CRAB and CRPA were continuously monitored via the hospital laboratory database and infection control surveillance records. Infection control measures to prevent transmission of CRAB and CRPA were implemented in the 2 units inclusive of an education program on infection control, a hand hygiene program, contact isolation, patient cohorting, environmental cleaning, and antimicrobial stewardship. Compliance with infection control processes, as well as antibiotic consumption, was continuously monitored, as described elsewhere. ${ }^{5}$ There was no routine hospital policy for active surveillance for CRAB and CRPA. For the outbreak investigation, a case patient was defined as a patient colonized or infected with CRAB and CRPA via clinical cultures procured more than 48 hours after admission to 1 of the 2 units. Hand hygiene compliance rate was defined as the number of observations that demonstrated compliance to hand hygiene before and after patient contact divided by the total number of observations. Compliance to contact isolation was defined as the number of observations confirming adherence to contact isolation precautions divided by the total number of observations. During environmental cleaning observations, the housekeeper was observed for cleaning of bed rails, overbed tables, infusion pumps, clean countertops, and soiled countertops, and results were recorded as "cleaned during observation," "not cleaned during observation," "not applicable," or "not observed." Weekly calculation of the fraction of items scored as "cleaned" versus "not cleaned" was recorded. Correlations between carbapenem consumption and CRAB or CRPA were performed using Pearson correlation analysis.

From October 1, 2012, to May 30, 2013 (periods 2-4), the incidence of CRPA steadily declined, while there was no significant change in the incidence of CRAB; compliance to infection control measures varied over time (Figure 1). The consumption of doripenem declined over the 11-month period, yet overall carbapenem consumption increased. There 